\title{
Overview on posterior reversible encephalopathy syndrome and pregnancy implications
}

\author{
Andreea Elena Dumitru' ${ }^{1}$ Ana Maria Vele ${ }^{1}$, Cristina Sciesser-Danciu², Corina Gica', Radu Botezatu ${ }^{1,3}$, \\ Nicolae Gica ${ }^{1,3}$, Gheorghe Peltecu ${ }^{1,3}$, Anca Marina Ciobanu', Anca Maria Panaitescu ${ }^{1,3}$ \\ ${ }^{1}$ Filantropia Clinical Hospital, Bucharest, Romania \\ 2"Dr. Constantin Opris" Emergency County Hospital, Baia Mare, Romania \\ 3"Carol Davila" University of Medicine and Pharmacy, Bucharest, Romania
}

\begin{abstract}
Posterior reversible encephalopathy syndrome (PRES) is a clinical radiologic syndrome characterized by visual deficits, altered consciousness, headache, confusion, seizures and focal neurological signs. Its pathophysiology is still uncertain, with vasogenic edema being the most frequent incriminated mechanism. It was described in correlation with hypertensive encephalopathy, eclampsia, use of cytotoxic and immunosuppressive drugs and renal diseases.

Regarding its association with pregnancy, it is important to deliver the baby as soon as possible, to administer proper medication for hypertension, with labetalol being first choice and combat seizures or any signs of status epilepticus.

A clear diagnosis can be established by combining clinical presentation with imaging techniques, especially T2 images of MRI. It has a rapid progression and good prognosis is obtained by early recognition and prompt treatment of associate condition.
\end{abstract}

Keywords: posterior reversible encephalopathy syndrome, vasogenic edema, seizure, neuroimaging, eclampsia

\section{INTRODUCTION}

Posterior reversible encephalopathy syndrome, also known as reversible posterior leukoencephalopathy syndrome (RPLS) is a radiographic syndrome of heterogeneous etiologies that are grouped together because of similar features associated with clinical manifestations such as visual deficits or blindness, altered consciousness, headache, confusion, seizures, focal neurological signs [1]. It might affect people under 2 years old or older than 60 and it was observed with increased frequency in women. Often the clinical context includes high blood pressure, pre/eclampsia, sepsis, impaired renal function, cytotoxic medication or autoimmune disease with laboratory data being modified by underlying conditions [2].

Pathophysiology is still unclear, but the most common mechanism explained is the vasogenic edema. Neuroimaging techniques by CT or MRI can establish the diagnosis. Prompt recognition and appropriate treatment can prevent the permanent damage and solve the clinical aspect within one week and the imaging modification over days to weeks [3].

\section{PATHOGENESIS}

The mechanisms of the disease are still unclear at the moment. Possible mechanisms are related to 
disturbed cerebral self-regulation, endothelial dysfunction [4] and hypertension [5].

The one based on endothelial dysfunction, present in the most underlying conditions such as eclampsia, sepsis, medication for cancer therapy and post-transplant status, autoimmune disorders, may have direct toxicity on vascular endothelium, producing capillary leakage and disruption of the blood-brain barrier, which may trigger vasogenic edema [6]. Moreover, while normal self-regulation involves constant maintenance of cerebral blood flow, when the cerebral self-regulation is exceeded, arterioles dilate and the flow increases in a pressure-passive manner with impact on systemic blood pressure. This results in brain hyperperfusion which may produce extravasation of fluid and blood components into parenchyma. In patients with chronic hypertension, vascular modifications alter the self-regulation to higher values of systemic blood pressure. As a result, patients with longstanding history of hypertension diagnosed with RPLS present with elevated blood pressures. Alternatively, in severe cases it was stated that disturbed self-regulation may produce focal vasoconstriction resulting in local hypoperfusion, cytotoxic edema or cerebral infarction $[7,8]$.

\section{CLINICAL ASPECTS}

Symptoms linked to reversible posterior leukoencephalopathy syndrome progress rapidly over hours to days. Patients may present with hypertension, but it is not mandatory and hypertensive crisis may precede the neurologic manifestations by 24 hours or more $[9,10]$. The clinical picture includes headaches (constant, nonlocalized, moderate to severe and unresponsive to treatment), altered consciousness (progressing from stupor to coma), visual disturbances (blurred vision), homonymous hemianopsia, or even cortical blindness; auras and seizures could be often the presenting manifestations [3]. Seizures and status epilepticus are common, while nonconvulsive status characterized by stereotypic movements such as staring, eye blinking, or head turning might be suspected in people with prolonged states of altered consciousness and confusion with postictal manifestation. Other symptoms less frequent are nausea, vomiting and brainstem deficits.

\section{PARTICULARITIES RELATED TO PREGNANCY}

Managing a case with PRES during pregnancy is similar to other medical condition associated, with recommendations to lower the elevated blood pressure, to treat any type of seizure, if present, and avoid specific chronic medication for epilepsy, with possible side effects. The aim of the management will be to deliver the fetus as soon as possible, control of hypertension with intravenous labetalol as the drug of choice and status epilepticus with magnesium sulphate at a similar dose as for preeclampsia or eclampsia (4 to 5 grams bolus intravenously, followed by an infusion of 1 to 2 grams per hour) [11].

Even though treatment is successful in the majority of cases, long-term sequelae have been seen in isolated patients. The most frequent consequences of PRES are seizures and/or epilepsy. One of the risk factors of developing long-term epilepsy is recurrent episodes of PRES. Ischemic or hemorrhage maternal strokes have also been published as a long term risk linked to PRES and has been thought to be induced by high blood pressure and high endothelial permeability most probably due to an inflammatory process developed in cytotoxic treatments. Nevertheless, pregnancy related hemodynamic changes (especially in eclampsia and preeclampsia) have also been demonstrated to cause vessel remodeling and an increase in bloodbrain barrier permeability. The vast majority of strokes developed during partum and few days after delivery.

\section{ESTABLISHING THE DIAGNOSIS}

While there are no specific criteria, clinicians should establish diagnosis by observing the clinical setting with extreme care for hypertension, immunosuppressive therapy and kidney diseases, combined with neurological examination. Neurologic symptoms of acute onset together with neuroimaging, in particular MRI-parietooccipital pattern, or, if possible, diffusion weighted imaging (DWI) may lead to PRES [12]. White matter ede$\mathrm{ma}$ in the posterior cerebral hemispheres is typical for PRES on neuroimaging [13]. Thus, MRI is the gold standard for the establishing the diagnosis with the $\mathrm{T} 2$ images revealing punctate or confluent 
symmetric bilateral hyperintensity in the parieto-occipital lobes, while the CT demonstrates symmetric hypodensities that involve the parietooccipital lobes of the brain $[14,15]$.

\section{DIFFERENTIAL DIAGNOSIS}

There is a broad spectrum for distinct differential diagnoses. Venous sinus thrombosis or subdural, subarachnoid or intracerebral hemorrhage may present the same clinical features. The suspicion of infectious encephalitis or meningitis, especially caused by herpes simplex, should be excluded. If it is confirmed it must be promptly treated with intravenous acyclovir.

Regarding the pathophysiology, another entity that needs to be excluded is the reversible cerebral vasoconstriction syndrome (RCVS). This presents thunderclap headaches accompanied or not by seizures or neurologic deficits and it is caused by multiple cerebral artery vasoconstriction with reversibility of symptoms and radiologic signs in approximately 3 months from the onset [5]. It is important to take into consideration the diagnosis of bilateral subacute posterior borders infarcts, whose prognosis and urgent treatment with thrombolysis differ from PRES. Hypoxic ischemic state, acute toxic leukoencephalopathy or postictal state may present similarities.

Imaging investigations can exclude also visual disturbances that appear during pregnancy (frequently associated with eclampsia/preeclampsia), such as bilateral serous retinal detachment (due to retinal vascular changes and choroidal perfusion), sudden visual loss due to central retinal artery occlusion, visual disturbance or even loss, related to postpartum HELLP syndrome or Sheehan syndrome.

\section{TREATMENT}

The management of such case includes intensive care unit (ICU) admission with continuous evaluation of consciousness impaired patients about need for upper airway protection, correction of electrolyte imbalance, especially hypoglycemia and vitamin B1 deficiency, anti-epileptic treatment and control of hypertension.

Treatment of PRES is targeting the underlying condition and the symptoms. The essential step of the treatment is to control the hypertension and maintain the blood pressure within normal range. The algorithm of choosing the antihypertensive treatment is based on general recommendations of cardiologist managing the hypertensive crisis. A reduction of blood pressure of $25 \%$ is recommended to control the damage. However, there is no proof that only the blood pressure alone can reverse the neurological damage and obtain a regression of clinical and radiological signs.

Anticonvulsive treatment is recommended to start from the first episode of seizures. However, the length of the treatment is still unclear. Some authors use it for 3 months after the resolution of symptoms while others stop it when there are no more MRI lesions and the symptoms disappeared.

Exposure to cytotoxic agents is a frequent cause of PRES. Controlling the trigger factor and the underlying cause is an important therapeutic step. Many immunosuppressive drugs like tacrolimus, cyclosporin, methotrexate, oxaliplatin, vincristine, cisplatin have been reported as the main factors of developing PRES, especially if corticosteroid treatment was also associated. The attitude is controversial, some authors recommending as the best option to discontinue and change the treatment while others recommending maintenance within the therapeutic level. Low magnesium level was found at the majority of patients with PRES and consequently, its level should be kept within the normal range.

\section{PREVENTION}

RPLS is usually reversible with prompt recognition and appropriate management. Treatment recommendations are based on the medical conditions presented.

In case of hypertension, a gradual decrease in blood pressure can dramatically improve the patient's outcome. In malignant hypertension, the aim is to reduce diastolic pressure to $100-105$ $\mathrm{mmHg}$, a more aggressive reduction being unnecessary because it may lead to cerebral, coronary and renal ischemia [16]. For patients with lower values of hypertension, lowering the values with 10 up to $25 \%$ is indicated. Effective medications for blood pressure reduction are nicardipine, labetalol and nitroprusside [17].

Reduction in drug dose or prompt removal of the cytotoxic or immunosuppressive drug is usual- 
ly recommended in cases of RPLS and is often associated with clinical improvement. When another immunosuppressive agent is substituted, patients must be followed closely for recurrence of RPLS. Performing routine neuroimaging is not recommended, but there should be a low threshold to pursue neuroimaging if symptoms arise suddenly or persist. Reviewing literature, it is certified that patients with mean blood pressure $>25 \%$ of baseline, creatinine levels $>1.8 \mathrm{mg} / \mathrm{dl}$ and fluid overload $>10 \%$ of baseline weight are at increased risk for PRES [18].

\section{CONCLUSIONS}

PRES syndrome should be taken into account when examining a pregnant woman with acute hypertension combined with epileptic seizures or neurologic disorders. Differential diagnosis is mandatory and the most important investigation is MRI to exclude other possible causes. Due to its association during pregnancy with eclampsia/preclampsia, special attention should be given to women diagnosed with acute hypertension more than for the ones with chronic disease. Prompt treatment with magnesium sulphate for epileptic status and controlled decrease of blood pressure values can control the symptoms and offer a good prognosis with complete remission.

Conflict of interest: none declared Financial support: none declared

\section{REFERENCES}

1. Hinchey J, Chaves C, Appignani B, et al. A reversible posterior leukoencephalopathy syndrome. N Engl J Med. 1996;334:494.

2. Fischer M, Schmutzhard E. Posterior reversible encephalopathy syndrome. J Neurol. 2017 Aug;264(8):1608-1616.

3. Roth $\mathrm{C}$, Ferbert A. The posterior reversible encephalopathy syndrome: what's certain, what's new? Pract Neurol. 2011;11:136-44.

4. Fugate JE, Rabinstein AA. Posterior reversible encephalopathy syndrome: clinical and radiological manifestations, pathophysiology, and outstanding questions. Lancet Neurol. 2015;14:914.

5. Levitt A, Zampolin R, Burns J, Bello JA, Slasky SE. Posterior Reversible Encephalopathy Syndrome and Reversible Cerebral Vasoconstriction Syndrome: Distinct Clinical Entities with Overlapping Pathophysiology. Radiol Clin North Am. 2019 Nov;57(6):1133-1146.

6. Christofidis C, Anastasiou A, Krasnikova E, Mandros C, Potolidis E. Posterior reversible encephalopathy syndrome as a complication of bacterial meningitis. Hippokratia. 2019;23(3):131-134.

7. Pilato F, Distefano M, Calandrelli R. Posterior Reversible Encephalopathy Syndrome and Reversible Cerebral Vasoconstriction Syndrome: Clinical and Radiological Considerations. Front Neurol. 2020 Feb 14;11:34.

8. Koch S, Rabinstein A, Falcone S, Forteza A. Diffusion-weighted imaging shows cytotoxic and vasogenic edema in eclampsia. AJNR Am J Neuroradiol. 2001;22:1068.

9. Omachi T, Shimo T, Nakano T, Takahashi M, Takaya J. Posterior reversible encephalopathy syndrome: pitfalls of T2 shine through in MRI. Clin Case Rep Rev. 2019;5.

10. Fischer M, Schmutzhard E. Posterior reversible encephalopathy syndrome. J Neurol. 2017;264(8):1608-1616.

11. Striano $P$, Striano S, Tortora F, et al. Clinical spectrum and critical care management of Posterior Reversible Encephalopathy Syndrome (PRES). Med Sci Monit. 2005;11:CR549.

12. Fugate JE, Rabinstein AA. Posterior reversible encephalopathy syndrome: clinical and radiological manifestations, pathophysiology, and outstanding questions. Lancet Neurol. 2015 Sep;14(9):914-925.

13. Cozzolino M, Bianchi C, Mariani G, Marchi L, Fambrini M, Mecacci F. Therapy and differential diagnosis of posterior reversible encephalopathy syndrome (PRES) during pregnancy and postpartum. Arch Gynecol Obstet. 2015 Dec;292(6):1217-23.

14. Doelken M, Lanz S, Rennert J, et al. Differentiation of cytotoxic and vasogenic edema in a patient with reversible posterior leukoencephalopathy syndrome using diffusion-weighted MRI. Diagn Interv Radiol. 2007;13:125.

15. Bartynski WS. Posterior reversible encephalopathy syndrome, part 1: fundamental imaging and clinical features. AJNR Am J Neuroradiol. 2008;29:1036.

16. Aronow WS. Treatment of hypertensive emergencies. Ann Transl Med. 2017;5(Suppl 1):S5.

17. Brathwaite L, Reif M. Hypertensive Emergencies: A Review of Common Presentations and Treatment Options. Cardiol Clin. 2019 Aug;37(3):275-286.

18. Tam CS, Galanos J, Seymour JF, Pitman AG, Stark RJ, Prince HM, Reversible posterior leukoencephalopathy syndrome complicating cytotoxic chemotherapy for hematologic malignancies. Am J Hematol. 2004;77(1):72. 